Pis'ma v ZhETF

\title{
Electronic Structure of New AFFeAs Prototype of Iron Arsenide Superconductors
}

\author{
I. A. Nekrasov ${ }^{+}$, Z. V. Pchelkina*, M. V. Sadovskii ${ }^{+}$ \\ + Institute for Electrophysics, Russian Academy of Sciences, Ural Division, 620016 Ekaterinburg, Russia \\ *Institute for Metal Physics, Russian Academy of Sciences, Ural Division, 620041 Ekaterinburg GSP-170, Russia \\ Submitted Today
}

\begin{abstract}
This work is provoked by recent discovery of new class prototype systems $\mathrm{AFFe} A \mathrm{~s}(\mathrm{~A}=\mathrm{Sr}, \mathrm{Ca})$ of novel layered ironpnictide High- $\mathrm{T}_{c}$ superconductors $\left(\mathrm{T}_{c}=36 \mathrm{~K}\right)$. Here we report ab initio LDA results for electronic structure of the AFFeAs systems. We provide detailed comparison between electronic properties of both new systems and reference LaOFeAs (La111) compound. In the vicinity of the Fermi level all three systems have essentially the same band dispersions. However for iron fluoride systems $\mathrm{F}(2 p)$ states were found to be separated in energy from $\operatorname{As}(4 p)$ ones in contrast to La111, where $\mathrm{O}(2 p)$ states strongly overlaps with $\operatorname{As}(4 p)$. Thus it should be more plausible to include only $\mathrm{Fe}(3 d)$ and $\mathrm{As}(4 p)$ orbitals into a realistic noninteracting model than for La111. Moreover Sr substitution with smaller ionic radius Ca in AFFeAs materials leads to a lattice contruction and stronger $\mathrm{Fe}(3 d)-\mathrm{As}(4 p)$ hybridization resulting in smaller value of the density of states at the Fermi level in the case of Ca compound. So to some extend Ca system reminds RE111 with later Rare Earths. However Fermi surface of new fluorides is found to be nearly perfect two-dimensional. Also we do not expect strong dependence of superconducting properties with respect to different types of A substitutes.
\end{abstract}

PACS: 74.25.Jb, 74.70.Dd, 71.20.-b, 74.70.-b

At the beginning of 2008 new layered ironpnictide 'High- $\mathrm{T}_{c}$ superconductors were found. Up to now there are known several classes of such systems. Following the 'order of discoveries: 1. Re111 ( $\mathrm{Re}=\mathrm{La}, \mathrm{Ce}, \mathrm{Pr}, \mathrm{Nd}, \mathrm{Sm})$ 'with parent compound $\mathrm{LaO}_{1-x} \mathrm{~F}_{x} \mathrm{FeAs}$ and $\mathrm{T}_{c}$ about 40-55 K 1, 2, 3, 4, 5, 6, 7, 8; ;. A122 (A=Ba, Sr) with parent system $\mathrm{Ba}_{1-x} \mathrm{~K}_{x} \mathrm{Fe}_{2} \mathrm{As}_{2}$ [9, 10, 11, 12, and $\mathrm{T}_{c}$ about $38 \mathrm{~K} ; 3 . \mathrm{Li}_{1-x} \mathrm{FeAs}$ with $\mathrm{T}_{c}=18 \mathrm{~K}[13,14$. 'Recently a new prototype material $\mathrm{SrFFeAs}$ was reported [15, 16]. This system has characteristic for ironarsenides SDW anomaly at about $175 \mathrm{~K}$. Later 'on this system doped with Co showed superconductivity at $\mathrm{T}_{c} \sim 5 \mathrm{~K}$ [17. Finally for the system $\mathrm{Sr}_{1-x} \mathrm{La}_{x} \mathrm{FFeAs}$ superconductivity with $\mathrm{T}_{c}=36 \mathrm{~K}$ was obtained by Zhu et al. 18. Also Zhu et al. 18, provide some crystallographic and resistivity data on CaFFeAs and EuFFeAs compounds. Inspired by this new step of ironpnictides developments we continue our work on these materials [19, 20, 21] and propose here first principle investigation of electronic structure of fluoride compounds.

LDA (local density approximation) calculated electronic structure of La111 was addressed in Refs. 23, 24, 25] and is related with one for $\mathrm{LaOFeP}$ [26]. Investigation of RE111 series showed band structure to be insensitive to the type of a Rare Earth [19]. Comparative study of electronic properties of other prototype systems $\mathrm{BaFe}_{2} \mathrm{As}_{2}$ 27, 28 and $\mathrm{LiFeAs}$ is presented in Refs. 20, 21].

The manuscript is organized as follows: first we describe crystal structure of ( $\mathrm{Sr}, \mathrm{Ca}) \mathrm{FFeAs}$ compounds and computational details. Then we provide circumstantial comparison between La111 [19] and both Sr and Ca compounds with respect to the LDA computed band dispersions, total, partial and orbitally resolved densities of states. Short summary finalize our paper.

\section{CRYSTALLOGRAPHIC AND COMPUTATIONAL DETAILS}

First crystal structure data for SrFFeAs were reported by Tegel et al. [15. As well as other FeAssystems [1, 29, 13. SrFFeAs has tetragonal structure with the space group $P 4 / \mathrm{nmm}$. Lattice parameters are $a=3.9930(1) \AA$ and $c=8.9546(1) \AA[15$. Atoms occupy following Wyckhoff positions [15, 22]: $\mathrm{F}(2 \mathrm{a})(0.75,0.25,0), \mathrm{Fe}(2 \mathrm{~b})(0.75,0.25,0.5)$ and $\mathrm{Sr}(2 \mathrm{c})$ $(0.25,0.25,0.1598), \mathrm{As}(2 \mathrm{c})(0.25,0.25,0.6527)$. This crystal structure was confirmed by several groups [16, 17, 18. Also Zhu et al. 18 determined lattice parameters of CaFFeAs as $a=3.879 \AA, c=8.601 \AA$. In Ref. [18. no analysis of atomic positions for Ca system was given. Thus for the time being in our calculations for Ca compound we use the same atomic positions as for $\mathrm{Sr}$ one. General appearance of the $\mathrm{Sr}(\mathrm{Ca}) \mathrm{FFeAs}$ crystal struc- 
ture is similar to one of La111 and so we refer reader to the Refs. [19, 20].

Main building blocks of new $\mathrm{Sr}$ and Ca systems are $\mathrm{AF}$ and FeAs tetrahedra layers with combined cation state " +1 " and " -1 " correspondingly. To this end AF layer is equivalent to the REO layer of RE111 systems [1. However larger ionic radius of $\mathrm{Sr}$ in comparison with, for example, La, gives sizeable expansion along $c$ axis of SrFFeAs structure, while $a$ and $b$ are almost not affected. Smaller ionic radii of Ca results in lattice contruction of $\mathrm{CaFFeAs}$ relative to $\mathrm{Sr}$ one. Nevertheless $c$ lattice parameter of CaFFeAs is of the same size as for PrOFeAs compound [8]. Physically important interatomic distances within $\mathrm{FeAs}_{4}$ tetrahedron layer Fe-Fe and Fe-As are practically identical to those of La111.

Linearized muffin-tin orbitals method (LMTO) 30] was employed to calculate electronic structure of $\mathrm{Sr}(\mathrm{Ca}) \mathrm{FFeAs}$ compounds.

\section{RESULTS AND DISCUSSION}

Upper panel of Fig. 1 shows comparison of band dispersions obtained within LDA for new SrFFeAs (black lines) compound with one recently published for La111 19] (gray lines). Bands crossing Fermi level are essentially identical for both compounds and belong to $\mathrm{Fe}(3 d)$ states (from $-2 \mathrm{eV}$ to $2.5 \mathrm{eV}$ ). Main difference comes in the region of $\mathrm{As}(4 p)$ states $(-5.5 \mathrm{eV}$ to $-2 \mathrm{eV})$. Here for La111 $\mathrm{As}(4 p)$ and $\mathrm{O}(2 p)$ states are essentially overlapped. For $\mathrm{SrFFeAs} \mathrm{As}(4 p)$ states stay still in interval $(-5.5 ;-2)$ eV but $\mathrm{F}(2 p)$ states are completely separated from them in energy and are located below between -7.5 and $-6 \mathrm{eV}$.

In the lower panel of Fig. 1 we compare band dispersions of Ca (gray lines) and Sr (black lines) iron fluorides. Band structure of both systems reminds each other. Again bands crossing Fermi level for Ca compound do not change much from those of $\mathrm{Sr}$ one. However because of lattice contruction and thus stronger hybridization effects $\mathrm{Fe}(3 d)$ band for $\mathrm{CaFFeAs}$ is slightly wider. For the same reason $\operatorname{As}(4 p)$ bands are slightly lower in energy than for $\mathrm{Sr}$ material. The $\mathrm{F}(2 p)$ bands are most affected by both lattice contruction and Sr to Ca substitution. First one leads to about $1 \mathrm{eV}$ broadening second one to the energy elevation. So after all $\mathrm{F}(2 p)$ bands somewhat intersect with $\mathrm{As}(4 p)$ bands. One can note that to some extend difference between $\mathrm{Sr}$ and $\mathrm{Ca}$ iron fluorides resembles very much difference between La111 and Pr111 iron arsenides (see Ref. [19]) although former one is larger and not that uniform.

Fig. 2 dispays direct matching of LDA calculated

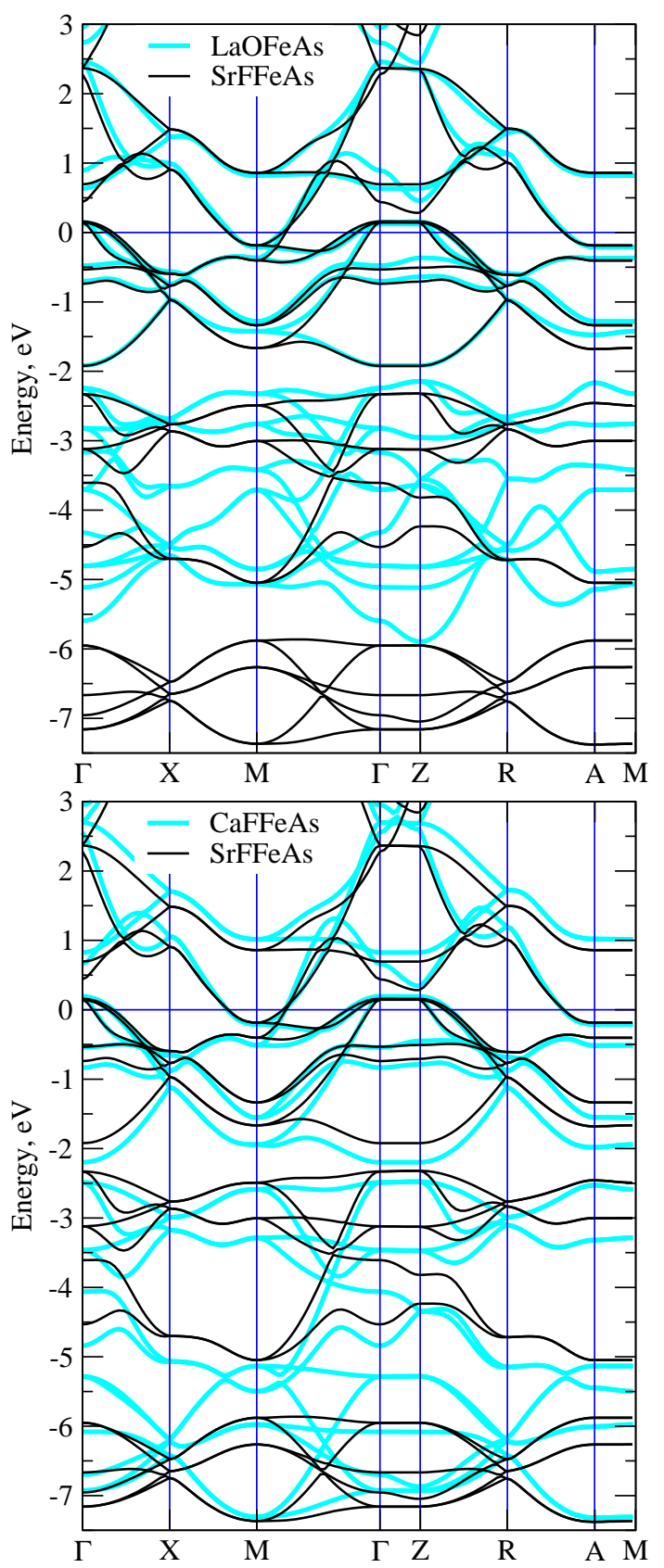

Fig. 1. Comparison of LDA band dispersions for La111 (wide gray lines) and SrFFeAs (black lines) on the upper panel and for CaFFeAs (wide gray lines) and SrFFeAs (black lines) on the lower panel. The Fermi level corresponds to zero.

DOS of Sr (solid black line) and Ca (dashed black line) iron fluorides with La111 (gray line) [19]. Upper panel contains total DOS of all three systems. The values of total DOS on the Fermi level are 3.65 state/eV/cell for CaFFeAs, 4.27 state/eV/cell for SrFFeAs and 4.01 state/eV/cell for reference La111 system, i.e. are only slightly different from each other. 
In the energy interval of $\mathrm{Fe}(3 d)$ states DOSes of all compounds are rather repetitive (see also next from top panel of Fig. 2). Moderate hybridization is observed between $\mathrm{Fe}(3 d)$ and $\operatorname{As}(4 p)$ states for both fluorides and practically no hybridization between $\mathrm{Fe}(3 d)$ and $\mathrm{F}(2 p)$ as well as between $\operatorname{As}(4 p)$ and $\mathrm{F}(2 p)$ states (see next from bottom panel of Fig. 2). For the $\mathrm{Sr}$ compound fluorine $2 p$ states are observed to be separated from all $\mathrm{Fe}(3 d)$ and $\mathrm{As}(4 p)$ states. And for the case of Ca system $\mathrm{F}(2 p)$ state touch $\mathrm{As}(4 p)$ ones.

Finally we present orbitally resolved $\mathrm{Fe}(3 d)$ DOS for $\mathrm{Sr}$ and $\mathrm{Ca}$ iron arsenide-fluorides in Fig. 3. Bird eye picture looks like the one for La111 (see Ref. [20]): Fermi level is crossed by bands of predominantly $t_{2 g}$ symmetry $-x z, y z, x^{2}-y^{2}$ (later because of $\pi / 4$ rotation of local coordinate system around $c$-axis). Nevertheless there are several fine distinctions with La111. The $x^{2}-y^{2}$ orbital is strongly changed around $0.5 \mathrm{eV}$. Some changes can be seen also for $3 z^{2}-r^{2}$ orbital around $-0.5 \mathrm{eV}$ and $x z, y z$ orbitals around $-1.5 \mathrm{eV}$ (see also Fig. 1, upper panel). The same is valid also for $\mathrm{Ca}$ system except some bands broadening. By looking on the Fig. 3 one can also understand lowering of the total density of states for Ca system with respect to $\mathrm{Sr}$ one mentioned above. Largest contribution to the DOS at the Fermi level comes from the $x^{2}-y^{2}$ orbital for all of FeAs systems [19, 20, 21. Because of stronger Fe-As hybridization it becomes wider and at the same time lower in intensity to keep normalization.

In Fig. 4 the Fermi surface (FS) of the new Sr fluoride system is compared with that of LaOFeAs 20 . Most important contrast to La111 FS is nearly perfect two-dimensional character of the FS of SrFFeAs. All cylinders are practically ideal especially two large ones around $\Gamma$-point. FS for Ca fluoride system is practically identical to that of $\mathrm{Sr}$ fluoride and we do not show it here.

\section{CONCLUSION}

LDA calculated electronic structure of new AFFeAs $(\mathrm{A}=\mathrm{Sr}, \mathrm{Ca})$ prototype of novel high- $\mathrm{T}_{c}$ ironpnitides superconductors is presented here in comparison with previously calculated [19, 20] for LaOFeAs material. Despite different chemical contents new systems and La111 have identical crystal structures. Thus in fluoride systems electronic properties are also determined by two-dimensional FeAs 4 layers as well. However LDA obtained Fermi surface of new fruorides is the most two-dimensional one among other known ironpnictides. Main distinction of fluoride systems from La111 is en-

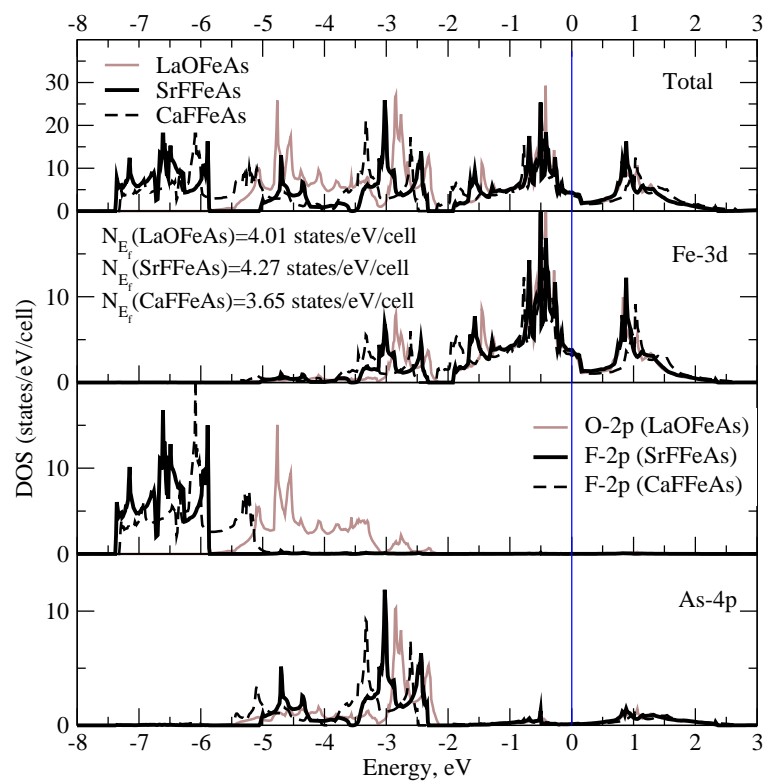

Fig. 2. Comparison of LDA calculated total and partial DOS for SrFFeAs (solid black line), CaFFeAs (dashed black line) and La111 [19] (gray line). Panels from top to bottom: total DOS, $\mathrm{Fe}(3 d), \mathrm{O}(2 p)$ and $\mathrm{F}(2 p), \operatorname{As}(4 p)$. The Fermi level corresponds to zero.

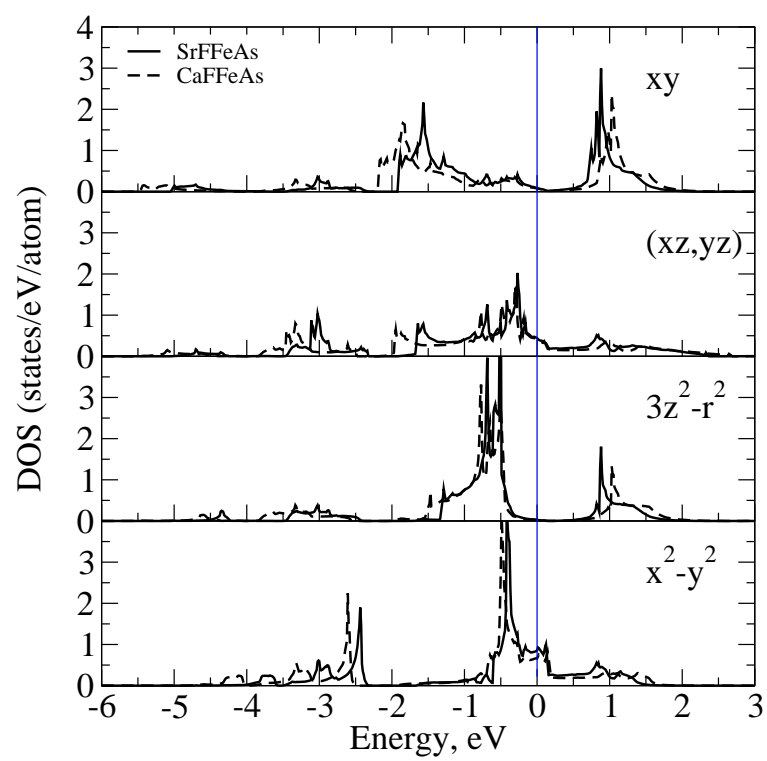

Fig. 3. Comparison of LDA orbitally resolved Fe-3d DOS for SrFFeAs (solid line) and CaFFeAs (dashed line). The Fermi level corresponds to zero.

ergy separation between $\mathrm{F}(2 p)$ state and $\operatorname{As}(4 p)$ states. Also practically no hybridization of $\mathrm{F}(2 p)$ and $\operatorname{As}(4 p)$ or $\mathrm{Fe}(3 d)$ states is observed. It makes non interacting realistic model of fluorides including only $\mathrm{As}(4 p)$ and $\mathrm{Fe}(3 d)$ states well justified and easier to construct. The CaF- 

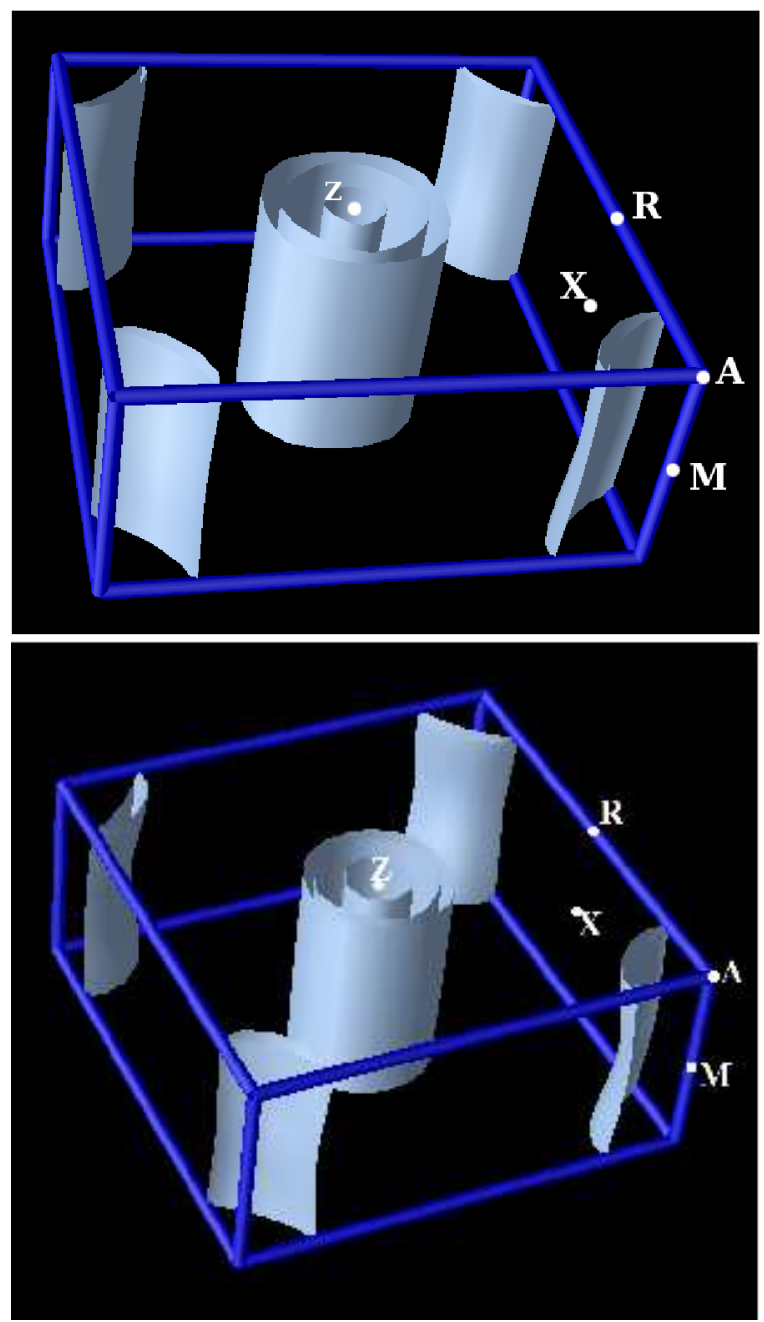

Fig. 4. Comparison of LDA calculated Fermi surfaces for SrFFeAs (upper panel) and LaOFeAs (lower panel).

FeAs with contructed lattice (since Ca ion is smaller) to some extend reminds RE111 systems with later RE. In this work we do not observe any significant difference of electronic structure of new fluorides and La111 close to the Fermi level, thus we do not expect any remarkable changes of superconducting properties with respect to different A substitutes. Lower values of DOS at the Fermi level for Ca compound probably makes it slightly less promising in this respect.

\section{ACKNOWLEDGEMENTS}

This work is supported by RFBR grants 0802-00021, 08-02-00712, RAS programs "Quantum macrophysics" and "Strongly correlated electrons in semiconductors, metals, superconductors and magnetic materials", Grants of President of Russia MK-2242.2007.2(IN), MK-3227.2008.2(ZP) and sci- entific school grant SS-1929.2008.2, interdisciplinary UB-SB RAS project, Dynasty Foundation (ZP) and Russian Science Support Foundation(IN). IN and MS thank University of Osaka (Japan) for hospitality during the visit in October 2008.

1. Y. Kamihara, T. Watanabe, M. Hirano, H. Hosono, J. Am. Chem. Soc. 130, 3296-3297 (2008).

2. G.F. Chen, Z. Li, G. Zhou, D. Wu, J. Dong, W.Z. Hu, P. Zheng, Z.J. Chen, J.L. Luo, N.L. Wang, Phys. Rev. Lett. 101, 057007 (2008).

3. X. Zhu, H. Yang, L. Fang, G. Mu, H.-H. Wen, Supercond. Sci. Technol. 21, 105001 (2008).

4. A.S. Sefat, M.A. McGuire, B.C. Sales, R. Jin, J.Y. Hove, D. Mandrus, Phys. Rev. B 77, 174503 (2008).

5. G. F. Chen, Z. Li, D. Wu, G. Li, W. Z. Hu, J. Dong, P. Zheng, J. L. Luo, N. L. Wang, Phys. Rev. Lett. 100, 247002 (2008).

6. X. H. Chen, T. Wu, G. Wu, R. H. Liu, H. Chen, D. F. Fang, Nature 453, 761 (2008).

7. Z.-A. Ren, J. Yang, W. Lu, W. Yi, X.-L Shen, G.-C. Che, L.-L. Sun, F. Zhou, Z.-X. Zhao, Europhys. Lett. 82, 57002 (2008).

8. Z.-A. Ren, J. Yang, W. Lu, W. Yi, G.-C. Che, X.-Li. Dong, L.-L. Sun, Z.-X. Zhao, Materials Research Innovations 12, 105 (2008).

9. M. Rotter, M. Tegel, D. Johrendt, Phys. Rev. Lett. 101, 107006 (2008).

10. G.F. Chen, Z. Li, G. Li, W.Z. Hu, J. Dong, X.D. Zhang, N.L. Wang, J.L. Luo, Chin. Phys. Lett. 25, 3403 (2008).

11. K. Sasmal, B. Lv, B. Lorenz, A. Guloy, F. Chen, Y. Xue, C.W. Chu, Phys. Rev. Lett. 101, 107007 (2008).

12. N. Ni, S.L. Bud'ko, A. Kreyssig, S. Nandi, G.E. Rustan, A.I. Goldman, S. Gupta, J.D. Corbett, A. Kracher, P.C. Canfield, Phys. Rev. B 78, 014507 (2008).

13. J.H. Tapp, Z. Tang, Bing Lv, K. Sasmal, B. Lorenz, Paul C.W. Chu, A, M. Guloy, Phys. Rev. B 78, 060505(R) (2008).

14. X.C.Wang, Q.Q. Liu, Y.X. Lv, W.B. Gao, L.X.Yang, R.C.Yu, F.Y.Li, C.Q. Jin, arXiv: 0806.4688.

15. M. Tegel, S. Johansson, V. Weiss, I. Schellenberg, W. Hermes, R. Poettgen, D. Johrendt, arXiv:0810.2120v1.

16. F. Han, X. Zhu, G. Mu, P. Cheng, H.H. Wen, arXiv:0810.2475v1.

17. S. Matsuishi, Y. Inoue, T. Nomura, M. Hirano, H. Hosono, arXiv:0810.2351 1.

18. X. Zhu, F. Han, P. Cheng, G. Mu, B. Shen, Hai-Hu Wen, arXiv:0810.2531 2 .

19. I.A. Nekrasov, Z.V. Pchelkina, M.V. Sadovskii. Pis'ma Zh. Eksp. Teor. Fiz. 87, 647 (2008) [JETP Letters 87 (2008)], arXiv: 0804.1239.

20. I.A. Nekrasov, Z.V. Pchelkina, M.V. Sadovskii, arXiv: arXiv:0806.2630, JETP Letters, 88, 144 (2008). 
21. I.A. Nekrasov, Z.V. Pchelkina, M.V. Sadovskii, arXiv:0807.1010, Pis'ma Zh. Eksp. Teor. Fiz. 88, 621 (2008).

22. For our computations lattice constants of $\mathrm{Sr}$ and $\mathrm{Ca}$ systems were taken from Ref. 18.

23. D.J. Singh, M.H. Du. Phys. Rev. Lett. 100, 237003 (2008).

24. L. Boeri, O.V. Dolgov, A.A. Golubov, Phys. Rev. Lett. 101, 026403 (2008).

25. I.I. Mazin, D.J. Singh, M.D. Johannes, M.H. Du, Phys. Rev. Lett. 101, 057003 (2008).

26. S. Lebègue, Phys. Rev. B 75, 035110 (2007).

27. I.R. Shein, A.L. Ivanovskii, arXiv: 0806.0750, Pis'ma Zh. Eksp. Teor. Fiz. 88, 115 (2008).

28. C. Krellner, N. Caroca-Canales, A. Jesche, H. Rosner, A. Ormeci, C. Geibel, Phys. Rev. B 78, 100504(R) (2008).

29. M. Rotter, M. Tegel, D. Johrendt, Phys. Rev. B 78, 020503(R) (2008).

30. O.K. Andersen. Phys. Rev. B 123060 (1975); O. Gunnarsson, O. Jepsen, O.K. Andersen. Phys. Rev. B 27 7144 (1983); O.K. Andersen, O. Jepsen. Phys. Rev. Lett. 532571 (1984). 